\title{
In Silico Docking Analysis of Poly Herbal Formulation Aadathodai Kudineer used in Siddha medicine in inhibiting Main Protease and ACE2 Receptor Spike protein SARS-CoV-2
}

\author{
Research Article
}

\section{Nikil Niva J1, Sasirekha R², Anbu N², Shree Devi M S4*, Sathiyarajeswaran P5}

\author{
1. PG Scholar, 2. Lecturer, Grade-II, 3. Professor, Head of the Department, Department of General Medicine, \\ Government Siddha Medical College, Chennai. \\ 4. Research Officer, 5. Director I/c, Siddha Central Research Institute, Chennai.
}

\begin{abstract}
Corona virus disease (COVID-19) is an infectious pandemic disease caused by the newly discovered novel corona virus. World Health Organization has declared the global health emergency due to COVID19 outbreak. Currently, there is no specific treatment or vaccine for fighting against this infectious disease. Aadathodai Kudineer is a drug indicated for Iya Erumal, Kozhai Kattu, Kabasuram. Upon the mortality and severity of the disease COVID19, we tried to identify the possible inhibition of phytocomponents of Aadathodai Kudineer in inhibiting Main Protease and ACE2 Receptor Spike protein SARS-CoV-2 through molecular docking studies. Methodology: In Silico molecular docking analysis was performed for phytocomponents present in the Aadathodai Kudineer formulation for targets main protease and ACE2 Receptor Spike protein, PDB ID: 6LU7 and PDB ID: 2AJF using Autodock tool. ADME properties was also predicted for all the above compounds. Results: Among the 9 active Phytocompounds present in the Aadathodai Kudineer formulation, Lupeol showed high binding affinity with COVID19 main protease and ACE2 receptor which shows the promising contrivance of protease inhibition. The ADME suggested that the formulation is free from toxic. Conclusion: The phytocomponents showed possible affinity towards these targets and has the lead molecules that inhibits COVID19 main protease and ACE2 receptor.
\end{abstract}

Key Words: Siddha formulation, Aadathodai Kudineer, SARS-CoV-2, COVID19, Molecular docking, ADME.

\section{Introduction}

On 31st December 2019, 27 cases of pneumonia of unknown aetiology were recognized in Wuhan City, Hubei area in China. The causative organism was recognized from throat swab tests led by the Chinese Centre for Disease Control and Prevention (CCDC) on seventh January 2020, and was along these lines named Severe Acute Respiratory Syndrome Coronavirus (SARS-CoV-2). The World Health Organization (WHO) named this infection as COVID-19. On 30th January 2020, the WHO proclaimed the Chinese episode of COVID-19 to be a Public Health Emergency of International Concern representing a high hazard to nations with weak wellbeing frameworks (1).

As of 27 July 2020, following data has been reported throughout the world, India \& Tamil Nadu. More than 16.4 million cases of COVID-19 have been reported in 185 countries and territories, resulting in more than 6,46,641 deaths. More than 9.51 million

* Corresponding Author:

\section{Shree Devi MS}

Research Officer, HoD, Department of Pharmacy,

Siddha Central Research Institute,

Chennai. 600 106,

Tamilnadu. India.

Email Id: shreemd@gmail.com people have recovered (2). The Ministry of Health and Family Welfare of India has confirmed a total of 14,35,453 cases, 9,17,567 recoveries (including 1 migration) and 32771 deaths in India. The Department of Health and Family Welfare of Tamil Nadu has confirmed a total of 2,13,723 cases, including 3493 deaths and 1,56,526 recoveries. Around 53703 active cases are reported (3).

The World Health Organization (WHO) welcomes innovations around the world including repurposing drugs, traditional medicines and developing new therapies in the search for potential treatments for COVID-19 (4). In China, traditional Chinese medicine is very useful to control and prevention and treatment for COVID 19 patients. In integrated approach is very success full treatment in COVID 19 patients in china (5).

Among six recognized streams of Indian Medicine System, Siddha medicine is one such traditional medicine originating in Tamil Nadu, India and practiced over centuries (6). Siddha system of medicine has played a major role in treating the diseases such as dengue, chikungunya. Both the TN Govt and union ministry of AYUSH has recommended an herbal siddha medicine called Nilavembu Kudineer as a treatment for dengue (7). This COVID 19 Pandemic Ministry of AYUSH publish the "Guidelines for Siddha Practitioners for COVID 19". 
Aadathodai Kudineer, a classical Siddha Formulation described in Siddha manuscript Gunapadam Mooligai Vaguppu and is used for Iya Erumal, Kozhai Kattu, Kabasuram. This poly herbal medicine contains three different ingredients such as Aadathodai, Kandankathiri, Seenthil (8). In Siddha treatment these three ingredients have been used extensively for the treatment of respiratory diseases such as cold, cough, whooping cough, chronic bronchitis, asthma, pneumonia and tuberculosis. Aadathodai has its own Anti-viral property (9), Antiasthmatic, Bronchodilator activity, Anti-tubercular activity, Anti-bacterial activity $(10-11)$, Antipyretic Activity (12). Seenthil has its own Antiviral property (13), Immunomodulatory activity (14), Anti-malarial, Anti-inflammatory, Antioxidant, Hepatoprotective, Immunomodulatory properties (15). Kandankathiri has it owns Anti-asthmatic activity, Anti-inflammatory activity, Cardio-protective activity (16), Antimicrobial activities, Anti-inflammatory activity, Anti-pyretic activity (17). Immunomodulatory activity, Anti-oxidant activity, Anti-pyretic activity, Hepatoprotective activity (18).

\section{Materials and methods}

Aadathodai Kudineer is a polyherbal Siddha formulation and active compounds in the formulation is indicated for Iya Erumal, Kozhai Kattu, Kabasuram. This formulation is having the following Siddha medicinal plants (Table 1).

Table 1: Aadathodai Kudineer their botanical name and its source.

\begin{tabular}{c|c|c|}
$\begin{array}{c}\text { S. } \\
\text { No }\end{array}$ & $\begin{array}{c}\text { Common } \\
\text { Name }\end{array}$ & $\begin{array}{c}\text { Botanical Name } \\
\text { Justicia adhatoda } \text { L }\end{array}$ \\
\hline 1 & Aadathodai & Solanum surattense BURM. \\
\hline 2 & Kandankathiri & F. \\
\hline 3 & Seenthil & $\begin{array}{c}\text { Tinospora } \\
\text { cordifolia } \text { (WILLD.) } \\
\text { HOOK.F. \& THOMS }\end{array}$ \\
\hline
\end{tabular}

\section{Protein ligand docking \\ Protein preparation}

The three-dimensional crystal structure of SARS-CoV-2 main protease (3-chymotrypsin-like protease (3CL pro) [PDB ID - 6LU7] and Angiotensinconverting enzyme 2 (ACE2) receptors - [PDB ID 2AJF] was retrieved from the Protein Data Bank (PDB) and energy minimized and then converted into their corresponding PDBQT formats.

\section{Ligand preparation}

The phytocomponents identified in Aadathodai Kudineer formulation such as Adhatodine, Anisotine, Berberine, Tinosporide, Apigenin, Diosgenin, Lupeol, Vasicinone, Vasicoline were selected for docking from the Aadathodai Kudineer formulation. Ligand molecules were downloaded from Pubchem (19) in sdf format. Optimization was done with the force field type
MMFF94 using Openbable softwares and saved as pdbqt format and used in docking studies.

\section{Molecular Docking}

Docking was performed with Autodock tool (20) a prevalent molecular screening tool for identifying binding energy between the 3D structures of each ligand and target proteins. Two target proteins PDB ID - 6LU7 and PDB ID - 2AJF were +9 selected, a Grid-free docking performed, and the binding energies of each ligand found.

\section{ADME properties prediction}

The ADME (absorption, distribution, metabolism, excretion and toxicity) properties of above mentioned phytocomponents predicted using SwissADME an online tool for ADME prediction (21).

\section{Results and Discussion}

Aadathodai Kudineer Chooranam is one among the poly herbal formulation used for the treatment of COVID-19, Molecular docking studies carried out for the nine phytocomponents present in Aadathodai Kudineer. SARS-CoV-2 use ACE2 receptors to gain entry to the target cells causing Corona Viral disease 19 in humans (22) and the main protease 3CLpro is highly essential for cleavage of polyprotein to get16 nonstructural proteins (called nsp1-nsp16). These nonstructural proteins are highly essential for viral replication. For this purpose, AutoDock4 docking program was employed, which uses Lamarckian genetic algorithm. The results of the binding affinity of these compound with their respective targets are given in (Table 2).

Table 2: Binding energy of various compounds involved in Aadathodai Kudineer

\begin{tabular}{|c|c|c|c|}
\hline $\begin{array}{c}\text { S. } \\
\text { No. }\end{array}$ & Compounds & $\begin{array}{c}\text { Binding Free } \\
\text { energy Kcal/ } \\
\text { mol } \\
\text { 3CLPRO }\end{array}$ & $\begin{array}{c}\text { Binding Free } \\
\text { energy Kcal/ } \\
\text { mol }\end{array}$ \\
\hline & & ACE2 \\
\hline 1 & Adhatodine & -7.47 & -5.13 \\
\hline 2 & Anisotine & -7.42 & -4.18 \\
\hline 3 & Berberine & -5.99 & -5.344 \\
\hline 4 & Tinosporide & -5.77 & -3.86 \\
\hline 5 & Apigenin & -4.55 & -2.23 \\
\hline 6 & Diosgenin & -7.96 & -6.27 \\
\hline 7 & Lupeol & -8.38 & -6.18 \\
\hline 8 & Vasicinone & -5.38 & -3.59 \\
\hline 9 & Vasicoline & -7.69 & -5.32 \\
\hline
\end{tabular}

Among all the docked compounds that showed a range of binding affinity Lupeol showed $8.38 \mathrm{kcal} /$ mol for 3CLPRO and $6.27 \mathrm{kcal} / \mathrm{mol}$ for ACE2 receptor respectively. Adhatodine, Anisotine, Tinosporide, Apigenin and Vasicoline possess $100 \%$ binding efficacy by interacting with both the core target amino acids (31 LYS and 353 LYS) present on the target. Followed by this other phytocompounds such as Berberine, Diosgenin, Lupeol and Vasicinone possess $50 \%$ affinity by binding with one of the target amino acid either with 
31 LYS or with 353 LYS present on the target receptor ACE-2. Anisotine has maximum of 6 interactions with the core active amino acid residues present on the target. Followed by this the compounds such as Adhatodine, Tinosporide and Lupeol ranked second with the maximum of 5 interactions. Similarly, the compounds Berberine, Apigenin and Vasicinone ranks third with the maximum of 4 interactions with the active site of the target enzyme 3CLpro. The ADME properties predicted using swissADME suggested that did not show any hepatocellular toxicity. The compound did not also show any blood-brain barrier crossing suggesting low toxicity induced upon intake. The compound is shows $90 \%$ solubility concentration of this phytocompound.

Table 3: Ligand Properties of the Compounds Selected for Docking Analysis

\begin{tabular}{|l|c|c|c|c|c|} 
Compound & Molar weight g/mol & Molecular Formula & H Bond Donor & $\begin{array}{l}\text { H Bond } \\
\text { Acceptor }\end{array}$ & $\begin{array}{l}\text { Rotatable } \\
\text { bonds }\end{array}$ \\
\hline Adhatodine & $337.416 \mathrm{~g} / \mathrm{mol}$ & $\mathrm{C}_{20} \mathrm{H}_{21} \mathrm{~N}_{3} \mathrm{O}_{2}$ & 1 & 2 & 4 \\
\hline Anisotine & $349.4 \mathrm{~g} / \mathrm{mol}$ & $\mathrm{C}_{20} \mathrm{H}_{19} \mathrm{~N}_{3} \mathrm{O}_{3}$ & 1 & 5 & 4 \\
\hline Berberine & $336.4 \mathrm{~g} / \mathrm{mol}$ & $\mathrm{C}_{20} \mathrm{H}_{18} \mathrm{NO}_{4}$ & 3 & 0 & 4 \\
\hline Tinosporide & $374.4 \mathrm{~g} / \mathrm{mol}$ & $\mathrm{C}_{20} \mathrm{H}_{22} \mathrm{O}_{7}$ & 1 & 7 & 1 \\
\hline Apigenin & $270.24 \mathrm{~g} / \mathrm{mol}$ & $\mathrm{C}_{15} \mathrm{H}_{10} \mathrm{O}_{5}$ & 3 & 5 & 0 \\
\hline Diosgenin & $414.6 \mathrm{~g} / \mathrm{mol}$ & $\mathrm{C}_{27} \mathrm{H}_{42} \mathrm{O}_{3}$ & 1 & 3 & 1 \\
\hline Lupeol & $426.7 \mathrm{~g} / \mathrm{mol}$ & $\mathrm{C}_{30} \mathrm{H}_{50} \mathrm{O}$ & 1 & 1 & 0 \\
\hline Vasicinone & $202.21 \mathrm{~g} / \mathrm{mol}$ & $\mathrm{C}_{11} \mathrm{H}_{10} \mathrm{~N}_{2} \mathrm{O}_{2}$ & 1 & 3 & 2
\end{tabular}

Table 4: Summary of the molecular docking studies of compounds against Angiotensin-converting enzyme 2 (ACE2) receptor- PDB $2 \mathrm{AJF}$

\begin{tabular}{|c|c|c|c|c|c|}
\hline Compounds & $\begin{array}{c}\text { Binding Free } \\
\text { energy Kcal/mol }\end{array}$ & $\begin{array}{c}\text { Inhibition constant } \\
\mathbf{K i} \boldsymbol{\mu M}(* \mathbf{m M}) \\
(* * \mathbf{n M})\end{array}$ & $\begin{array}{c}\text { Electrostatic } \\
\text { energy Kcal/mol }\end{array}$ & $\begin{array}{c}\text { Intermolecular } \\
\text { energy Kcal/mol }\end{array}$ & $\begin{array}{c}\text { Interaction } \\
\text { Surface }\end{array}$ \\
\hline Adhatodine & -5.13 & 172.73 & -0.82 & -5.04 & 530.48 \\
Anisotine & -4.18 & 867.69 & -0.06 & -5.03 & 503.55 \\
Berberine & -5.344 & 103.4 & -1.14 & -4.88 & 551.99 \\
\hline nosporide & -3.86 & $1.47^{*}$ & -0.16 & -4.62 & 444.65 \\
\hline Apigenin & -2.23 & 23.08 & -0.12 & -4.05 & 430.24 \\
\hline Dosgenin & -6.27 & 25.36 & -0.02 & -6.57 & 582.39 \\
\hline peol & -6.18 & 29.59 & -0.16 & -6.77 & 655.34 \\
\hline aicinone & -3.59 & $2.34^{*}$ & -0.17 & -3.89 & 344.17 \\
\hline sicoline & -5.32 & 126.28 & -0.60 & -5.23 & 463.48
\end{tabular}

Table 5: Amino acid Residue Interaction of Lead against Angiotensin-converting enzyme 2 (ACE2) receptor- PDB 2AJF

\begin{tabular}{|c|c|c|c|c|c|c|c|c|}
\hline Molecule & Interactions & \multicolumn{7}{|c|}{ Amino Acid Residue- Binding } \\
\hline Adhatodine & 2 & 31 LYS & $34 \mathrm{HIS}$ & $35 \mathrm{GLU}$ & $37 \mathrm{GLU}$ & $38 \mathrm{ASP}$ & 353 LYS & - \\
\hline Anisotine & 2 & 31 LYS & 34 HIS & $35 \mathrm{GLU}$ & $37 \mathrm{GLU}$ & 38 ASP & 353 LYS & - \\
\hline Berberine & 1 & 33 ASN & 34 HIS & $37 \mathrm{GLU}$ & 38 ASP & 353 LYS & 389 PRO & 393 ARG \\
\hline Tinosporide & 2 & 31 LYS & 34 HIS & $35 \mathrm{GLU}$ & $37 \mathrm{GLU}$ & 38 ASP & 353 LYS & - \\
\hline Apigenin & 2 & 31 LYS & 34 HIS & 38 ASP & 353 LYS & & & - \\
\hline Diosgenin & 1 & $33 \mathrm{ASN}$ & 34 HIS & 37 GLU & 38 ASP & 353 LYS & 389 PRO & 393 ARG \\
\hline Lupeol & 1 & 30 ASP & 33 ASN & 34 HIS & 37 GLU & 353 LYS & 389 PRO & $393 \mathrm{ARG}$ \\
\hline Vasicinone & 1 & 31 LYS & 34 HIS & $35 \mathrm{GLU}$ & 38 ASP & - & - & - \\
\hline Vasicoline & 2 & 31 LYS & 34 HIS & $35 \mathrm{GLU}$ & 37 GLU & 38 ASP & 353 LYS & - \\
\hline
\end{tabular}

Table 6: Summary of the molecular docking studies of compounds against COVID-19 main protease (3-chymotrypsin-like protease (3CL pro) - PDB 6LU7

\begin{tabular}{|c|c|c|c|c|c|}
\hline Compounds & $\begin{array}{c}\text { Binding Free } \\
\text { energy Kcal } / \mathrm{mol}\end{array}$ & $\begin{array}{c}\text { Inhibition constant } \\
\text { Ki } \underset{\mu M(* m M)}{(* * n M)}\end{array}$ & $\begin{array}{c}\text { Electrostatic } \\
\text { energy Kcal/mol }\end{array}$ & $\begin{array}{l}\text { Intermolecular } \\
\text { energy Kcal/mol }\end{array}$ & $\begin{array}{c}\text { Total } \\
\text { Interaction } \\
\text { Surface }\end{array}$ \\
\hline hatodine & -7.47 & 3.36 & -0.36 & -8.29 & 766.16 \\
\hline isotine & -7.42 & 3.66 & -0.03 & -8.11 & 790.62 \\
\hline berine & -5.99 & 40.33 & -0.17 & -6.71 & 726.25 \\
\hline osporide & -5.77 & 59.28 & -0.07 & -6.59 & 758.09 \\
\hline igenin & -4.55 & 45.50 & -0.21 & -6.39 & 650.78 \\
\hline sgenin & -7.96 & 1.46 & -0.18 & -8.29 & 832.88 \\
\hline peol & -8.38 & $716.67 * *$ & -0.03 & -8.98 & 911.38 \\
\hline sicinone & -5.38 & 113.14 & -0.10 & -5.68 & 493.23 \\
\hline sicoline & -7.69 & 2.31 & -0.32 & -7.61 & 687.35 \\
\hline
\end{tabular}


Table 7: Amino acid Residue Interaction of Lead against

COVID-19 main protease (3-chymotrypsin-like protease (3CL pro) - PDB 6LU7

Molecule Interactions

\begin{tabular}{|c|c|c|c|c|c|c|c|c|c|c|c|}
\hline 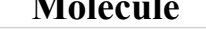 & Miceractions & \multicolumn{10}{|c|}{ Р⿴囗十 } \\
\hline Adhatodine & 5 & $\begin{array}{c}41 \\
\text { HIS }\end{array}$ & $\begin{array}{c}49 \\
\text { MET }\end{array}$ & $\begin{array}{c}54 \\
\text { TYR }\end{array}$ & $\begin{array}{c}142 \\
\text { ASN }\end{array}$ & $\begin{array}{r}144 \\
\text { SER }\end{array}$ & $\begin{array}{c}145 \\
\text { CYS }\end{array}$ & $\begin{array}{l}163 \\
\text { HIS }\end{array}$ & $\begin{array}{c}165 \\
\text { MET }\end{array}$ & $\begin{array}{c}166 \\
\text { GLU }\end{array}$ & $\begin{array}{c}189 \\
\text { GLN }\end{array}$ \\
\hline Anisotine & 6 & $\begin{array}{c}27 \\
\text { LEU }\end{array}$ & $\begin{array}{c}41 \\
\text { HIS }\end{array}$ & $\begin{array}{c}144 \\
\text { SER }\end{array}$ & $\begin{array}{c}145 \\
\text { CYS }\end{array}$ & $\begin{array}{l}163 \\
\text { HIS }\end{array}$ & $\begin{array}{r}165 \\
\text { MET }\end{array}$ & $\begin{array}{c}166 \\
\text { GLU }\end{array}$ & 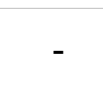 & 20 & 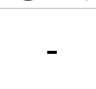 \\
\hline Berberine & 4 & $\begin{array}{c}41 \\
\text { HIS }\end{array}$ & $\begin{array}{c}49 \\
\text { MET }\end{array}$ & $\begin{array}{c}145 \\
\text { CYS }\end{array}$ & $\begin{array}{c}165 \\
\text { MET }\end{array}$ & $\begin{array}{c}166 \\
\text { GLU }\end{array}$ & $\begin{array}{r}189 \\
\text { GLN }\end{array}$ & C & - & - & - \\
\hline Tinosporide & 5 & $\begin{array}{c}41 \\
\text { HIS }\end{array}$ & $\begin{array}{c}142 \\
\text { ASN }\end{array}$ & $\begin{array}{c}144 \\
\text { SER }\end{array}$ & $\begin{array}{c}145 \\
\text { CYS }\end{array}$ & $\begin{array}{l}163 \\
\text { HIS }\end{array}$ & $\begin{array}{r}165 \\
\text { MET }\end{array}$ & $\begin{array}{c}166 \\
\text { GLU }\end{array}$ & $\begin{array}{c}189 \\
\text { GLN }\end{array}$ & - & - \\
\hline Apigenin & 4 & $\begin{array}{c}41 \\
\text { HIS }\end{array}$ & $\begin{array}{c}49 \\
\text { MET }\end{array}$ & $\begin{array}{c}52 \\
\text { PRO }\end{array}$ & $\begin{array}{c}54 \\
\text { TYR }\end{array}$ & $\begin{array}{c}145 \\
\text { CYS }\end{array}$ & $\begin{array}{l}163 \\
\text { HIS }\end{array}$ & $\begin{array}{c}166 \\
\text { GLU }\end{array}$ & $\begin{array}{c}189 \\
\text { GLN }\end{array}$ & - & - \\
\hline Diosgenin & 3 & $\begin{array}{c}25 \\
\text { THR }\end{array}$ & $\begin{array}{c}41 \\
\text { HIS }\end{array}$ & $\begin{array}{c}49 \\
\text { MET }\end{array}$ & $\begin{array}{c}143 \\
\text { GLY }\end{array}$ & $\begin{array}{c}145 \\
\text { CYS }\end{array}$ & $\begin{array}{c}166 \\
\text { GLU }\end{array}$ & $\begin{array}{r}189 \\
\text { GLN }\end{array}$ & - & - & - \\
\hline Lupeol & 5 & $\begin{array}{c}41 \\
\text { HIS }\end{array}$ & $\begin{array}{c}142 \\
\text { ASN }\end{array}$ & $\begin{array}{c}145 \\
\text { CYS }\end{array}$ & $\begin{array}{c}165 \\
\text { MET }\end{array}$ & $\begin{array}{c}166 \\
\text { GLU }\end{array}$ & $\begin{array}{r}168 \\
\text { PRO }\end{array}$ & $\begin{array}{c}189 \\
\text { GLN }\end{array}$ & - & - & - \\
\hline Vasicinone & 4 & $\begin{array}{c}27 \\
\text { LEU }\end{array}$ & $\begin{array}{c}41 \\
\text { HIS }\end{array}$ & $\begin{array}{l}144 \\
\text { SER }\end{array}$ & $\begin{array}{c}145 \\
\text { CYS }\end{array}$ & $\begin{array}{l}163 \\
\text { HIS }\end{array}$ & & - & - & - & - \\
\hline Vasicoline & 3 & $\begin{array}{r}165 \\
\text { MET }\end{array}$ & $\begin{array}{c}166 \\
\text { GLU }\end{array}$ & $\begin{array}{c}167 \\
\text { LEU }\end{array}$ & $\begin{array}{l}168 \\
\text { PRO }\end{array}$ & $\begin{array}{c}189 \\
\text { GLN }\end{array}$ & $\begin{array}{c}192 \\
\text { GLN }\end{array}$ & - & - & - & - \\
\hline
\end{tabular}

Table 8: In silico pharmacokinetics properties of phytochemical constituents of Aadathodai Kudineer

\begin{tabular}{|l|c|c|c|c|c|}
$\begin{array}{c}\text { Phytochemical } \\
\text { constituent }\end{array}$ & $\begin{array}{c}\text { Intestinal } \\
\text { absorption }\end{array}$ & $\begin{array}{c}\text { BBB } \\
\text { permeability }\end{array}$ & $\begin{array}{c}\text { Human Vd } \\
(\mathbf{L} / \mathbf{k g})\end{array}$ & $\begin{array}{c}\text { Total clearance (mg/kg/ } \\
\text { day) }\end{array}$ & $\begin{array}{c}\text { Renal OCT2 } \\
\text { substrate }\end{array}$ \\
\hline Adhatodine & 86.22 & -0.127 & 0.08 & 0.58 & No \\
\hline Anisotine & 94.97 & -0.381 & -0.092 & 0.675 & No \\
\hline Berberine & 97.147 & 0.198 & 0.58 & 1.27 & No \\
\hline Tinosporide & 100 & -0.482 & 0.15 & 0.414 & No \\
\hline Apigenin & 93.25 & -0.734 & 0.822 & 0.566 & Yes \\
\hline Diosgenin & 96.565 & 0.2 & 0.426 & 0.328 & No \\
\hline Lupeol & 95.782 & 0.726 & 0 & 0.153 & No \\
\hline Vasicinone & 92.532 & -0.206 & 0.142 & 0.568 & Yes
\end{tabular}

Table 9: The predicted pharmacokinetics properties of phytochemical constituents for Cytochrome Inhibition and P-glycoprotein studies for Aadathodai Kudineer

\begin{tabular}{|c|c|c|c|c|}
\hline $\begin{array}{l}\text { Phytochemical } \\
\text { constituent }\end{array}$ & $\begin{array}{c}\text { CYP2D6 and } \\
\text { CYP3A4 } \\
\text { substrate }\end{array}$ & $\begin{array}{l}\text { CYP enzymes } \\
\text { inhibition }\end{array}$ & P-gp substrate & P-gp I or II inhibition \\
\hline Adhatodine & No & CYP1A2 & No & No \\
\hline Anisotine & CYP3A4 & $\begin{array}{c}\text { CYP1A2 } \\
\text { CYP2C19 } \\
\text { CYP2C9 } \\
\text { CYP3A4 }\end{array}$ & substrate & P-gp I and II \\
\hline Berberine & CYP3A4 & $\begin{array}{l}\text { CYP1A2 } \\
\text { CYP2D6 } \\
\text { CYP3A4 }\end{array}$ & substrate & P-gp II \\
\hline Tinosporide & CYP3A4 & No & No & No \\
\hline Apigenin & No & $\begin{array}{c}\text { CYP1A2 } \\
\text { CYP2C19 }\end{array}$ & substrate & No \\
\hline Diosgenin & CYP3A4 & No & No & P-gp I and II \\
\hline Lupeol & CYP3A4 & No & No & P-gp I and II \\
\hline Vasicinone & No & CYP1A2 & substrate & No \\
\hline Vasicoline & $\begin{array}{l}\text { CYP3A4 } \\
\text { CYP2D6 }\end{array}$ & $\begin{array}{c}\text { CYP1A2 } \\
\text { CYP2C19 } \\
\text { CYP2C9 } \\
\text { CYP2D6 }\end{array}$ & No & No \\
\hline
\end{tabular}


Table 10: The predicted toxicity of phytochemical constituents of Aadathodai Kudineer

\begin{tabular}{|c|c|c|c|c|c|c|c|c|}
\hline $\begin{array}{l}\text { Phytochem } \\
\text { ical } \\
\text { constituent }\end{array}$ & $\begin{array}{c}\text { AMES } \\
\text { tox. }\end{array}$ & $\begin{array}{c}\text { hERG I } \\
\text { or II } \\
\text { inhibition }\end{array}$ & $\begin{array}{c}\text { Hepatoto } \\
\text { xicity }\end{array}$ & $\begin{array}{c}\text { Skin } \\
\text { sensitizati } \\
\text { on }\end{array}$ & $\begin{array}{l}\text { Carcinog } \\
\text { enicity }\end{array}$ & $\begin{array}{c}\text { Human } \\
\text { maximu } \\
\text { m } \\
\text { tolerated } \\
\text { dose } \\
\text { (mg/kg/ } \\
\text { day) }\end{array}$ & $\begin{array}{l}\text { Oral rat } \\
\text { acute } \\
\text { toxicity } \\
\text { (mol/ } \\
\text { kg) }\end{array}$ & $\begin{array}{c}\text { Oral rat } \\
\text { chronic } \\
\text { tox. } \\
\text { (mg/ } \\
\text { kg_bw/ } \\
\text { day) }\end{array}$ \\
\hline Adhatodine & Yes & No & No & No & No & 0.15 & 1.956 & 2.564 \\
\hline Anisotine & $\mathrm{NO}$ & NO & Yes & No & No & 0.158 & 2.268 & 1.758 \\
\hline Berberine & Yes & No & Yes & No & No & 0.144 & 2.571 & 1.89 \\
\hline Tinosporide & No & No & Yes & No & No & -0.373 & 3.165 & 0.967 \\
\hline Apigenin & No & No & No & No & No & 0.328 & 2.45 & 2.2983 \\
\hline Diosgenin & No & hERG II & No & No & No & -0.559 & 1.921 & 1.452 \\
\hline Lupeol & No & hERG II & No & No & No & -0.502 & 2.563 & 0.89 \\
\hline Vasicinone & No & No & No & No & No & 0.332 & 1.91 & 1.708 \\
\hline Vasicoline & Yes & hERG II & Yes & No & No & 0.088 & 2.621 & 0.728 \\
\hline
\end{tabular}

Figure 1a: 3D- Structure of Angiotensin-converting enzyme 2 (ACE2) receptor- PDB ID 2AJF

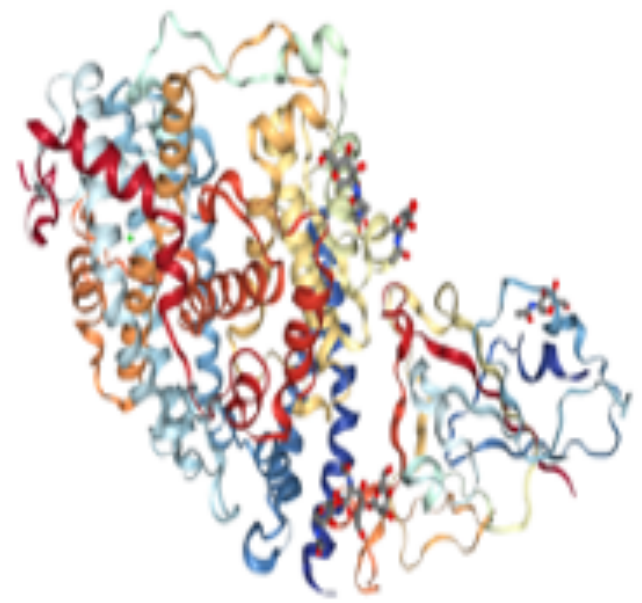

Figure 1b: 3D crystalline structure of the target protein COVID-19 main protease (3-chymotrypsinlike protease (3CL pro) - PDB ID 6LU7

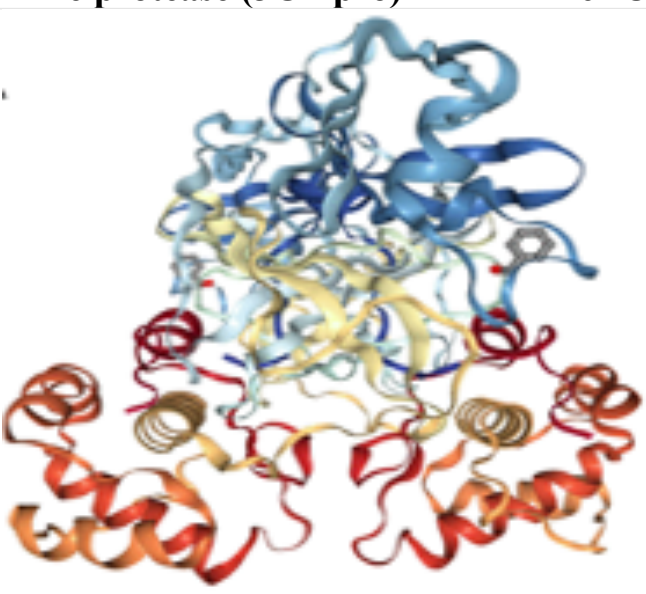

Figure 2: 2D and 3D Structure of Selected Ligands
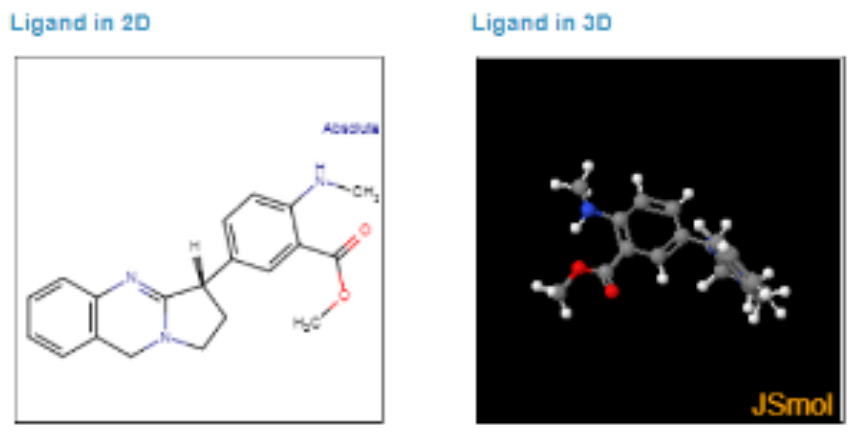
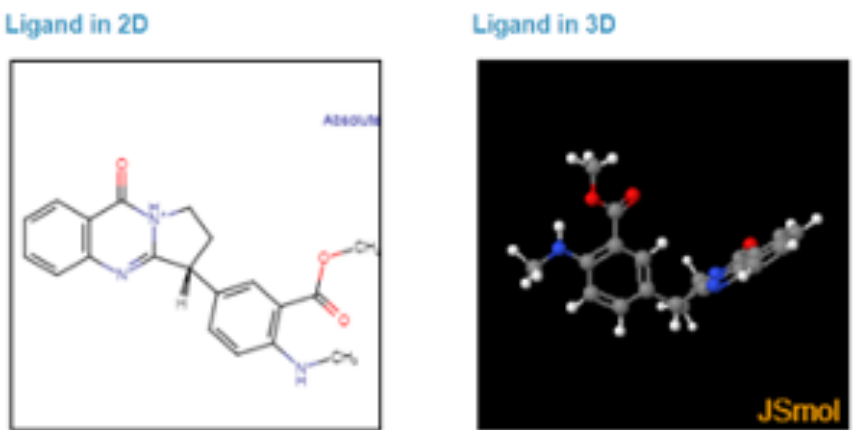

Figure 2a : Adhatodine

Figure 2b : Anisotine 

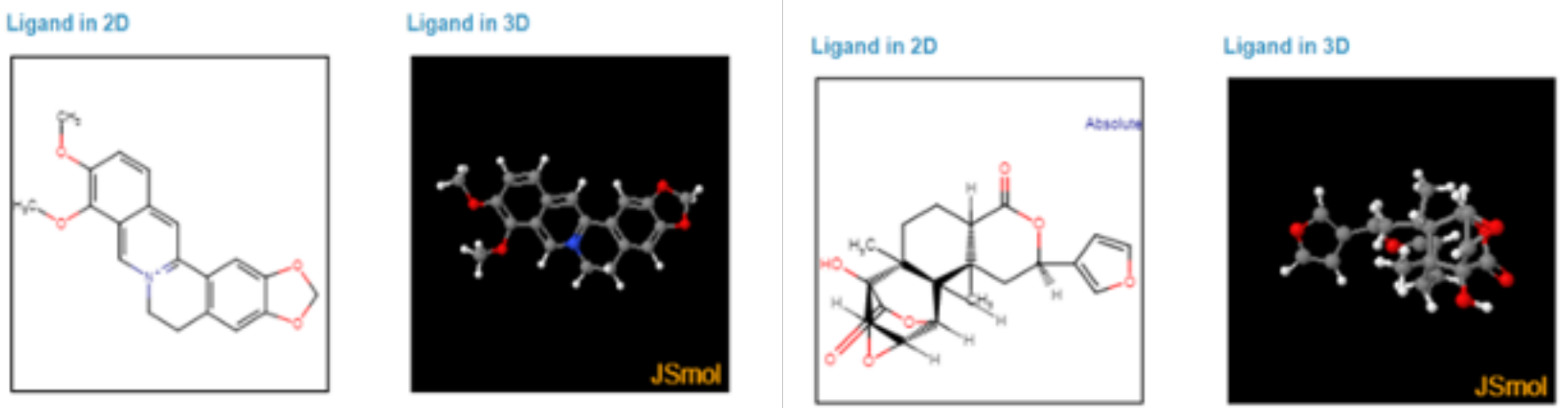

Figure 2c : Berberine
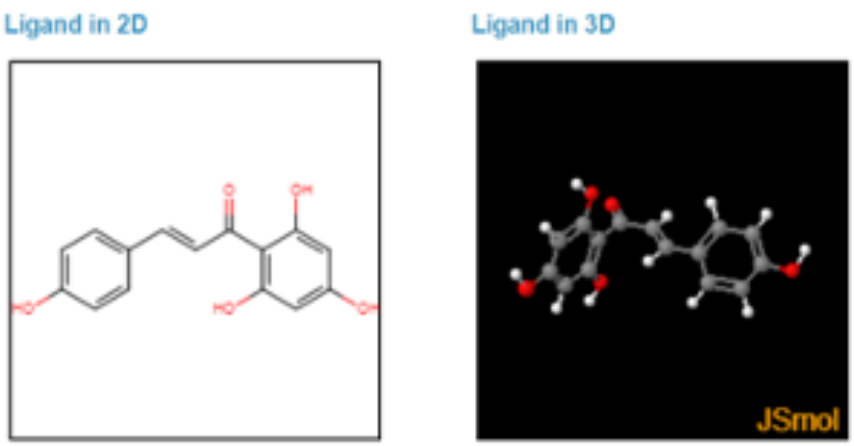

Figure 2e : Apigenin

Ligand in 2D
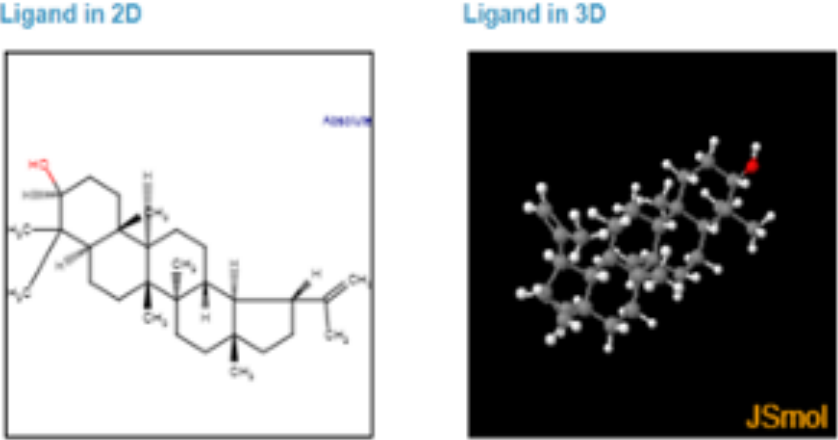

Figure 2g : Lupeol

Ligand in $2 \mathrm{D}$

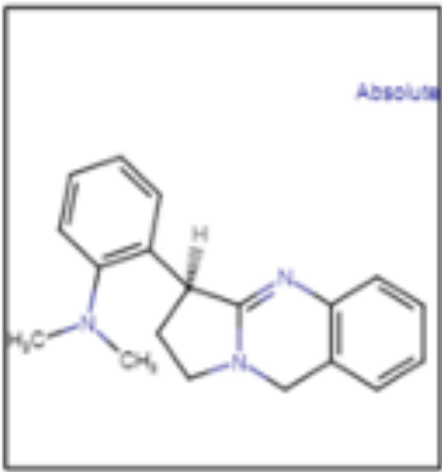

Figure 2d : Tinosporide
Ligand in 20

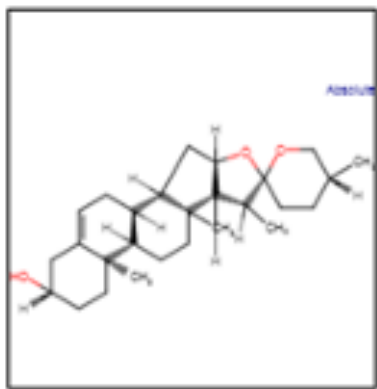

Ligand in 3D

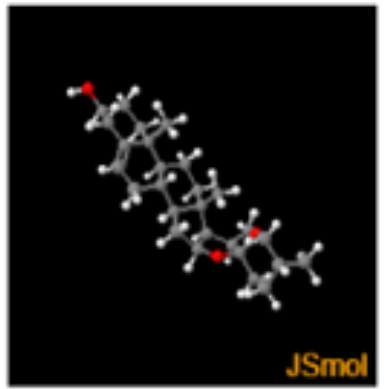

Figure 2f : Diosgenin

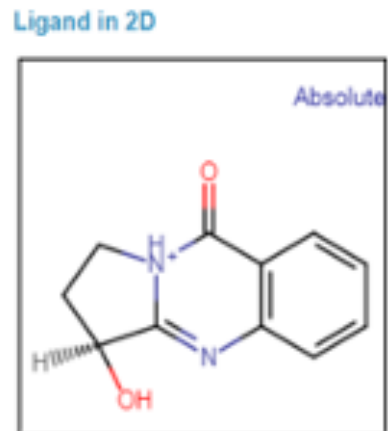

Ligand in 30

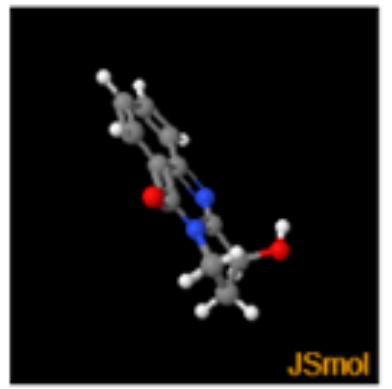

Figure 2h : Vasicinone

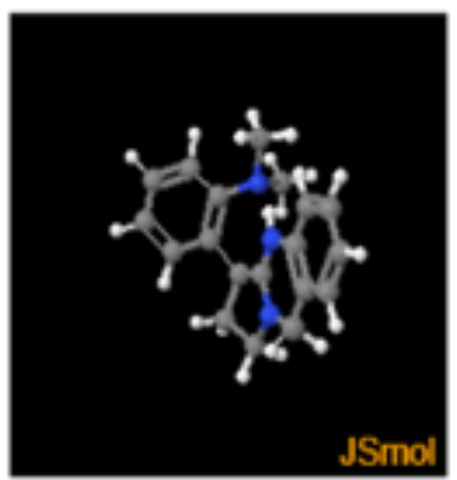

Figure 2i : Vasicoline 


\section{Conclusion}

Various Phytoconstituents of the Aadathodai Kudineer Chooranam such as Adhatodine, Anisotine, Tinosporide, Apigenin, Vasicoline, Berberine, Diosgenin, Lupeol and Vasicinone present in the herbs of the formulation Aadathodai Kudineer revels significant binding against the target protein thereby it was concluded that these compounds may exerts promising inhibiting against ACE-2 receptor and hereby halt the host-viral interface and exerts promising inhibiting against 3 CL pro enzyme and hereby halt the formation of 16 non-structural proteins (nsp1-nsp16) that are highly essential for viral replication and there by prevents the viral survival in the host environment. This formulation have high binding affinities to the C3like protease and ACE2 Receptor Spike protein of COVID-19 and can possibly be future therapeutics against this coronavirus if in silico studies are confirmed with antiviral activity studies. It remains a possibility that this formulation on treatment may contribute to the lower incidences of COVID-19 cases in India. Further, preclinical and clinical trials have to be conducted in order to know the exact mechanism and efficacy of Aadathodai Kudineer in SARS-CoV-2.

\section{Conflict of Interest}

Nil.

\section{Funding Information}

Nil.

\section{References}

1. Sohrabi C, Alsafi Z, O’Neill N, Khan M, Kerwan A, Al-Jabir A, Iosifidis C, Agha R. World Health Organization declares global emergency: A review of the 2019 novel coronavirus (COVID-19). International Journal of Surgery. $2020 \mathrm{Feb} 26$.

2. Covid19. who.int. 2020. WHO Coronavirus Disease (COVID-19) Dashboard. [online] Available at: $<$ https://covid19.who.int/> [Accessed 28 July 2020].

3. MyGov.in. 2020. \#Indiafightscorona COVID-19. [online] Available at: <https://www.mygov.in/ covid-19> [Accessed 28 July 2020].

4. WHO | Regional Office for Africa. 2020. WHO Supports Scientifically-Proven Traditional Medicine. [online] Available at: <https:// www. afro.who.int/news/who-supports scientifically-proven-traditional-medicine? gclid=EAIaIQobChMIm4eL25rv6gIVWCUrCh2Pu QqmEAAYASAAEgKF7PD_BwE> [Accessed 28 July 2020].

5. Ni L, Yuan W, Chen L, Han C, Zhang H, Luan X, Zhao Y, Xu J, Chen H. Combating COVID-19 with integrated traditional Chinese and Western medicine in China. Acta Pharmaceutica Sinica B. 2020 Jun 27.

6. Muthiah K, Ganesan K, Ponnaiah M, Parameswaran S. Concepts of body constitution in traditional Siddha texts: A literature review. Journal of Ayurveda and integrative medicine. 2019 Apr 1;10(2):131-4.

7. Jain J, Kumar A, Narayanan V, Ramaswamy RS, Sathiyarajeswaran P, Devi MS, Kannan M, Sunil S. Antiviral activity of ethanolic extract of Nilavembu Kudineer against dengue and chikungunya virus through in vitro evaluation. Journal of Ayurveda and integrative medicine. 2019.

8. Murukesa Muthaliyar K S, Gunapadam Mooligai Vaguppu, 9th edition, Dept Of Indian Medicine Homeopathy Chennai. 2013,Page no - 65 .

9. Chavan R, Chowdhary A. In vitro inhibitory activity of Justicia adhatoda extracts against influenza virus infection and hemagglutination. Int. J. Pharm. Sci. Rev. Res. 2014;25(2):231-6.

10. Chavan R, Gohil D, Shah VI, Kothari S, Chowdhary AS. Antiviral activity of Indian medicinal plant Justicia Adhatoda against herpes simplex virus: an in-vitro study. Int J Pharm Bio Sci. 2013;4(4).

11. Gangwar AK, Ghosh AK. Medicinal uses and pharmacological activity of Adhatoda vasica. International journal of herbal medicine. 2014;2(1):88-91.

12. Belemkar S, Thakre SA, Pata MK. Evaluation of anti-inflammatory and analgesic activities of methanolic extract of Adhatoda vasica Nees and Mentha piperita Linn. Inventi Rapid Ethnopharmacol. 2013.1;2:1-6.

13. Purthvish R, Gopinatha SM. Antiviral prospective of Tinospora cordifolia on HSV-1. Int. J. Curr. Microbiol. App. Sci. 2018.7(1):3617-24.

14. Sachan S, Dhama K, Latheef SK, Abdul Samad H, Mariappan AK, Munuswamy P, Singh R, Singh KP, Malik YS, Singh RK. Immunomodulatory Potential of Tinospora cordifolia and $\mathrm{CpG}$ ODN (TLR21 Agonist) against the Very Virulent, Infectious Bursal Disease Virus in SPF Chicks. Vaccines. 2019 Sep;7(3):106.

15. Joshi G, Kaur R. Tinospora cordifolia: a phytopharmacological review. International journal of Pharmaceutical sciences and research. 2016 Mar $1 ; 7(3): 890$

16. Tekuri SK, Pasupuleti SK, Konidala KK, Amuru SR, Bassaiahgari P, Pabbaraju N. Phytochemical and pharmacological activities of Solanum surattense Burm. f.-A review. Journal of Applied Pharmaceutical Science. 2019 Mar;9(03):126-36.

17. Patel K, Singh RB, Patel DK. Medicinal significance, pharmacological activities, and analytical aspects of solasodine: A concise report of 
current scientific literature. Journal of Acute Disease. 2013 Jan 1;2(2):92-8.

18. Arora P, Ansari S H, Phyto-Pharmacological Review of Solanum xanthjocarpum Schrad and Wendl, International Journal of Pharmacognosy and Phytochemical Research 2019, 11(4);244-249.

19. Ihlenfeldt WD. PubChem. In: Applied Chemoinformatics, 2018, 245-258. [https://doi.org/ 10.1002/9783527806539.ch6e].

20. Morris G. M., D. S. Goodsell, et al. Automated docking using a Lamarckian genetic algorithm and an empirical binding free energy function. Journal of Computational Chemistry 19 (14), 1639-1662(1998).

21. A. Daina, O. Michielin, and V. Zoete. (2017). "SwissADME: A free web tool to evaluate pharmacokinetics, drug- likeness and medicinal chemistry friendliness ofsmall molecules," Sci. Rep., vol. 7.

22. Alexandra C. Walls,1,5 Young-Jun Park,1,5 M. (2020). Alejandra Tortorici,1,2 Abigail Wall,3 Andrew T. McGuire,3,4 and David Veesler, Structure, Function, and Antigenicity of the SARSCoV-2 Spike Glycoprotein, Cell, Volume 181, Issue 2.pages 281-292.e6. 